Received: 19 August 2016

Accepted: 5 May 2017

Published online: 21 June 2017

\section{Bounds for coherence of quantum superpositions in high dimension}

\author{
Qiu-Ling Yue ${ }^{1,2}$, Fei Gao ${ }^{1}$, Qiao-Yan Wen ${ }^{1}$ \& Wei-Wei Zhang ${ }^{3}$
}

Quantum coherence plays a major role in the promotion for quantum information processing and designing quantum technology. Since coherence is rooted in superposition principle, it is vital to understand the coherence change with respect to superpositions. Here we study the bounds for coherence of quantum superpositions in high dimension. We consider three most frequently used measures of coherence, $i$.e. the relative entropy of coherence, $l_{1}$ norm of coherence and robustness of coherence. For a quantum state (an arbitrary dimension) and its arbitrary decomposition, we give the upper and lower bounds for coherence of the superposition state in terms of the coherence of the states being superposed.

Quantum coherence is a fundamental feature of quantum mechanics. As one of the most crucial physical resources, it plays a primary role in quantum information processing ${ }^{1-3}$, computational task $\mathrm{k}^{4,5}$, quantum metrol$\mathrm{ogy}^{6,7}$, thermodynamics ${ }^{8-10}$, and quantum biology ${ }^{11,12}$. The theory of coherence as a resource was first set forth in ref. 13, where the authors introduced a rigorous framework for the quantification of coherence and identified the computable measures of coherence. From resource-driven viewpoint, there is a growing number of work studying coherence including different coherence measures ${ }^{14-18}$, the properties of coherence ${ }^{19,20}$, the freezing phenomenon of coherence $e^{21,22}$, the relation among coherence, entanglement and quantum correlation ${ }^{23-25}$, and so on refs $26,27$.

Despite coherence derives from the superposition of states, the coherence of a superposition state cannot be directly deduced from the coherence of the individual states being superposed. We illustrate it with the following examples: given a state

$$
\left|\Omega_{1}\right\rangle=\frac{1}{\sqrt{2}}(|0\rangle+|1\rangle),
$$

it is obvious that the coherence of $|0\rangle,|1\rangle$ is 0 , while the coherence of $\left|\Omega_{1}\right\rangle$ reaches maximum value. Here, we consider coherence in the computational basis. In the following, we show an opposite example. Given a state

$$
\left|\Omega_{2}\right\rangle=\frac{1}{\sqrt{2}}(|+\rangle+|-\rangle)
$$

where $| \pm\rangle=(|0\rangle \pm|1\rangle) / \sqrt{2}$. Interestingly, under computational basis the coherence of $|+\rangle,|-\rangle$ reaches maximum value while the coherence of their superposition $\left|\Omega_{2}\right\rangle$ is 0 .

Our concern here is that: given an arbitrary state $|\Omega\rangle$ and its arbitrary decomposition

$$
|\Omega\rangle=\alpha|\Phi\rangle+\beta|\Psi\rangle,
$$

what is the relation between the coherence of $|\Omega\rangle$ and the coherence of $|\Phi\rangle$ and $|\Psi\rangle$ ? Because of the importance of coherence in quantum physics and superposition for coherence, the solutions for this problem will provide a theoretical foundation for potential applications of quantum resource and quantum information processing.

Similar problem has been studied in the field of entanglement. In 2006, Linden et al. firstly studied the relation between the entanglement of $|\Omega\rangle$ and the entanglement of $|\Phi\rangle$ and $|\Psi\rangle^{28}$, in which they gave the upper bounds on the entanglement of the superposition state in terms of the entanglement of the states being superposed using von Neumann entropy of the reduced state as a measure of entanglement. Thereafter, there are some related works.

${ }^{1}$ State Key Laboratory of Networking and Switching Technology, Beijing University of Posts and Telecommunications, Beijing, 100876, China. ${ }^{2}$ State Key Laboratory of Information Security, (Institute of Information Engineering, Chinese Academy of Sciences), Beijing, 100093, China. ${ }^{3}$ Hefei National Laboratory for Physical Sciences at Microscale, University of Science and Technology of China, Hefei, Anhui, 230026, China. Correspondence and requests for materials should be addressed to F.G. (email: gaofei_bupt@hotmail.com) 
Ref. 29 gave a tighter upper bound on the same question and also gave a lower bound. Refs 30, 31 studied the same problem considering concurrence (another measure of entanglement) and gave the corresponding upper and lower bound. However, this problem is still open in the field of coherence where only two specific cases have been discussed ${ }^{32,33}$.

In this work, we analyze the relation between the coherence of $|\Omega\rangle$ and the coherence of its decomposition. We systematically study this problem considering three kinds of coherence measure, and give the corresponding tight upper and lower bounds. Our results can be used for estimating the coherence range of the superposition state. For example, given the coherence of two states, we do not even need to know what the state is, we can estimate the range of the coherence resource we can get from their superposition state. In addition, armed with these relationships on coherence of superpositions, we can easily monitor the coherence change in the quantum information processing, such as coherence distillation. Coherence is likewise a measure of information carrying ability. The more coherence, the more information can be carried in the states.

In our work, we focus on orthogonal version of problem (i.e. $|\Phi\rangle$ and $|\Psi\rangle$ are orthogonal states). We can get the non-orthogonal version of the problem easily by the following decomposition,

$$
|\Omega\rangle=\alpha|\Phi\rangle+\beta|\Psi\rangle=\alpha|\Phi\rangle+\beta\left(\gamma|\Phi\rangle+\sqrt{1-\gamma}\left|\Phi^{\perp}\right\rangle\right),
$$

where $|\Phi\rangle$ and $\left|\Phi^{\perp}\right\rangle$ are orthogonal states.

\section{Results}

Relative entropy of coherence. A well-defined and frequently used coherence measure is the relative entropy of coherence, which is proposed and studied in ref. 13. With a particular entropic formula, the relative entropy of coherence has some clear physical meanings, such as it is equal to the optimal distillation rate for standard coherence distillation ${ }^{34}$, and can also be interpreted as the minimal amount of noise required for fully decohering the state ${ }^{27,35}$. In this section, we study the relationship between the coherence of two orthogonal states and the coherence of its decomposition using the relative entropy of coherence.

Given a particular basis $\left\{|i\rangle_{i=1}^{n}\right\}$, the definition of relative entropy of coherence ${ }^{13}$ is

$$
C_{r e}(\rho)=S\left(\rho_{d}\right)-S(\rho),
$$

where $\rho$ is density operator and $\rho_{d}$ denotes the state obtained from $\rho$ by deleting all off-diagonal elements under the particular basis, and $S(\rho)$ is von Neumann entropy of $\rho$. In the case of a pure state $|\phi\rangle$, its relative entropy of coherence can be expressed as

$$
C_{r e}(\phi)=S\left(|\phi\rangle_{d}\langle\phi|\right) .
$$

In the following, we will give the bounds with respect to relative entropy of coherence. The proof of this Theorem is in Methods.

Theorem 1. Given two orthogonal states $|\Phi\rangle,|\Psi\rangle$, and two complex number $\alpha, \beta$ satisfying $\| \alpha|\Phi\rangle+\beta|\Psi\rangle \|=1$, the coherence of the superposition $|\Omega\rangle=\alpha|\Phi\rangle+\beta|\Psi\rangle$ satisfies

$$
C_{r e}(\Omega) \leq \min \left\{\begin{array}{l}
\frac{1}{q} f(p), \\
f(p)+2(1-q)|\alpha||\beta| \log (n-1)+(1-q),
\end{array}\right.
$$

for $0<p<1$, where

$$
q=\frac{p(1-p)}{(1-p)|\alpha|^{2}+p|\beta|^{2}}, f(p)=p C_{r e}(\Phi)+(1-p) C_{r e}(\Psi)+h_{2}(p)
$$

$h_{2}(x) \equiv-x \log x-(1-x) \log (1-x)$, and $n$ is the dimension of Hilbert space.

Remark 1: The result in ref. 32 is the case with $p=|\alpha|^{2}$ in Theorem 1 .

Remark 2: We give an example to show the upper bound in Theorem 1 is tight in some cases.

Example 1: Consider the following case:

$$
\begin{aligned}
|\Phi\rangle & =\frac{1}{\sqrt{2}}\left(|1\rangle+\frac{1}{\sqrt{n-1}}(|2\rangle+|3\rangle+\cdots+|n\rangle)\right), \\
|\Psi\rangle & =\frac{1}{\sqrt{2}}\left(|1\rangle-\frac{1}{\sqrt{n-1}}(|2\rangle+|3\rangle+\cdots+|n\rangle)\right), \\
\alpha & =-\beta=\frac{1}{\sqrt{2}}
\end{aligned}
$$

where $n$ is the dimension of Hilbert space.

In this case, we can see that the upper bound in Theorem 1 is tight. Considering $p=|\alpha|^{2}$, the first bound in Eq. (7) equals to $2\left[|\alpha|^{2} C_{r e}(\Phi)+|\beta|^{2} C_{r e}(\Psi)+h_{2}\left(|\alpha|^{2}\right)\right]$. The coherence of $|\Phi\rangle$ and $|\Psi\rangle$ both equal to $\frac{1}{2} \log (n-1)+1$, and the coherence of their superposition state $|\Omega\rangle=\alpha|\Phi\rangle+\beta|\Psi\rangle$ is $\log (n-1)$. The ratio of $C_{r e}(\Omega)$ and $|\alpha|^{2} C_{r e}(\Phi)+|\beta|^{2} C_{r e}(\Psi)+h_{2}\left(|\alpha|^{2}\right)$ converges to 2 when $n$ is infinite as shown in Eq. (9). 

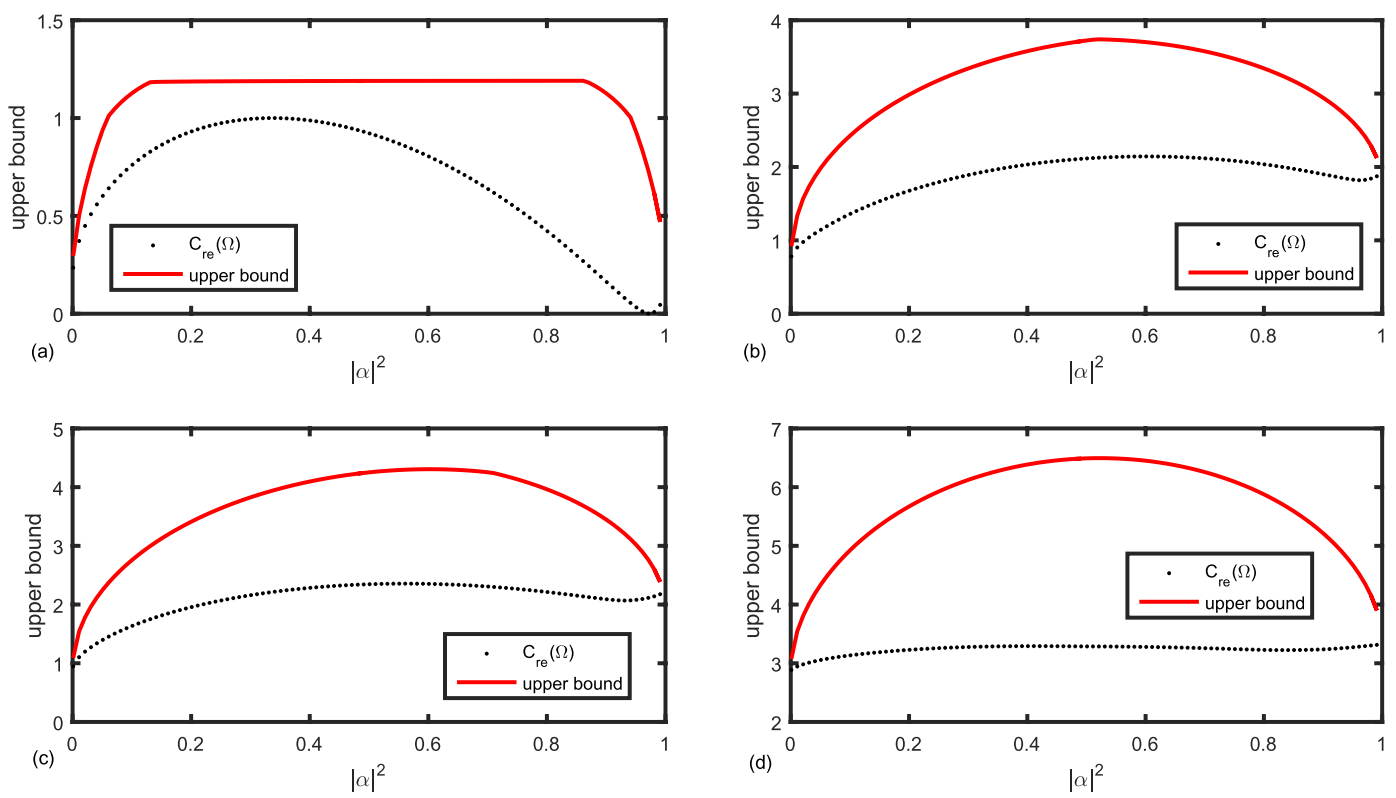

Figure 1. The upper bounds on coherence of the superpositions for dimension $=2,5,7,15$, with $|\Phi\rangle$ and $|\Psi\rangle$ as defined in the text. The black dotted line is the exact value of $C_{r e}(\Omega)$; the red solid one is the upper bound in Theorem 1. Note that both $\alpha$ and $\beta$ are chosen to be positive numbers here.

$$
\lim _{n \rightarrow \infty} \frac{C_{r e}(\Omega)}{2\left[|\alpha|^{2} C_{r e}(\Phi)+|\beta|^{2} C_{r e}(\Psi)+h_{2}\left(|\alpha|^{2}\right)\right]}=1 .
$$

We give numerical simulations for the upper bound in Theorem 1 and the exact coherence of $|\Omega\rangle$ in different dimensions by choosing two random orthogonal states $|\Phi\rangle$ and $|\Psi\rangle$ as shown in Fig. 1. The sub-figures (a-d) represent the comparison in different dimensions $(2,5,7,15$ respectively). Let $|\Phi\rangle$ and $|\Psi\rangle$ are chosen randomly as following: (a) $|\Phi\rangle=0.9863|0\rangle-0.1650|1\rangle$, and $|\Psi\rangle=0.1650|0\rangle+0.9863|1\rangle$; (b) $|\Phi\rangle=0.6252|0\rangle-0.4357|1\rangle$ $-0.1846|2\rangle-0.5545|3\rangle+0.2788|4\rangle$, and $|\Psi\rangle=0.2079|0\rangle+0.1082|1\rangle+0.9322|2\rangle-0.0226|3\rangle+0.2750|4\rangle$; (c) $|\Phi\rangle=-0.2503|0\rangle+0.1711|1\rangle+0.2273|2\rangle+0.6442|3\rangle+0.2732|4\rangle+0.5262|5\rangle+0.2998|6\rangle$, a n d $|\Psi\rangle=0.9139|0\rangle+0.0736|1\rangle+0.2927|2\rangle+0.0612|3\rangle-0.0641|4\rangle+0.2555|5\rangle-0.0224|6\rangle ;(\mathrm{d})|\Phi\rangle=0.3266|0\rangle$ $+0.1666|1\rangle-0.1765|2\rangle+0.2515|3\rangle+0.4549|4\rangle+0.1634|5\rangle-0.2075|6\rangle-0.2327|7\rangle+0.0535|8\rangle-0.1524|9\rangle$ $-0.4818|10\rangle+0.1975|11\rangle+0.0855|12\rangle-0.2922|13\rangle-0.2244|14\rangle$, and $|\Psi\rangle=0.1829|0\rangle+0.4304|1\rangle+0.0664|2\rangle+$ $0.0460|3\rangle+0.1304|4\rangle+0.3446|5\rangle+0.0927|6\rangle+0.0213|7\rangle+0.1006|8\rangle+0.4598|9\rangle+0.0060|10\rangle+0.0322|11\rangle$ $+0.3234|12\rangle+0.2864|13\rangle+0.4698|14\rangle$.

The black dotted line is the exact coherence of superposition state $|\Omega\rangle$, the red solid one is the upper bound in Theorem 1. Note that $\alpha$ and $\beta$ we chose are both positive numbers. From this figure, we can see that given the state $|\Phi\rangle$ and $|\Psi\rangle$, the upper bound in Theorem 1 depends on the parameter $\alpha$. Parallelly, given the value of $\alpha$, the coherence of states $|\Phi\rangle$ and $|\Psi\rangle$ also affects upper bound in Theorem 1.

Now, we move to analyze the lower bounds. By constructing a special state and measuring it with an incoherent operation, we can get the lower bound for coherence of superpositions considering relative entropy of coherence as stated in following theorem. Its detailed proof is in Methods.

Theorem 2. Given two orthogonal states $|\Phi\rangle,|\Psi\rangle$, and two complex number $\alpha, \beta$ satisfying $\| \alpha|\Phi\rangle+\beta|\Psi\rangle \|=1$, the coherence of the superposition $|\Omega\rangle=\alpha|\Phi\rangle+\beta|\Psi\rangle$ satisfies

$$
\begin{aligned}
C_{r e}(\Omega) \geq & \max \left\{t C_{r e}(\Phi)-\frac{1-p}{p} C_{r e}(\Psi)\right. \\
& \left.-\frac{1}{p} h_{2}(p), t \in\left\{\frac{(1-p)|\alpha|^{2}}{1-p|\alpha|^{2}}, \frac{(1-p)|\beta|^{2}}{1-p|\beta|^{2}}\right\}\right\},
\end{aligned}
$$

for $0<p<1$, and $_{2}(x) \equiv-x \log x-(1-x) \log (1-x)$.

$l_{1}$ norm coherence. Another measure of coherence, $l_{1}$ norm, is defined with the off-diagonal elements of the considered quantum state. This definition is intuitive for the measure of coherence and satisfies the necessary properties presented in ref. 13 . 


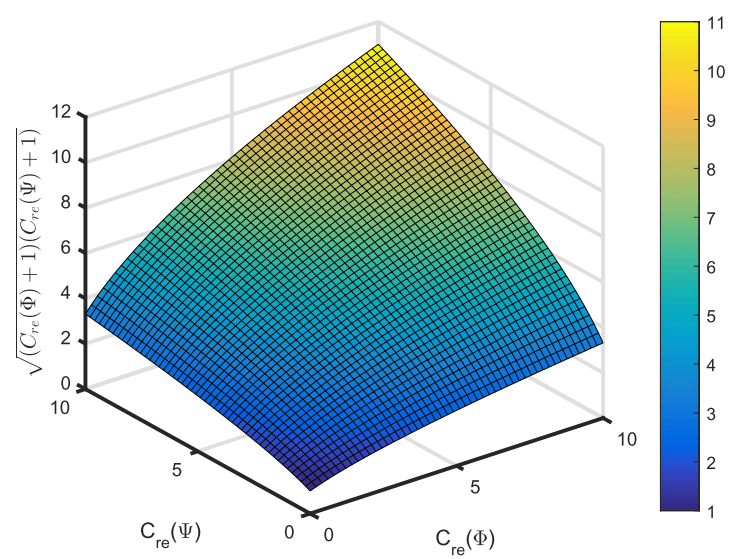

Figure 2. The comparison between $\sqrt{\left(C_{l_{1}}(\Phi)+1\right)\left(C_{l_{1}}(\Psi)+1\right)}$ and $n-1$. Here the domains of $C_{l_{1}}(\Phi)$ and $C_{l_{1}}(\Psi)$ are set as $[0,10]$.

Given a fixed basis $\left\{|i\rangle_{i=1}^{n}\right\}$, where $n$ is the dimension of Hilbert space. The definition of $l_{1}$ norm of coherence of a state $\rho$ is ref. 13

$$
C_{l_{1}}(\rho) \equiv \sum_{i \neq j}\left|\rho_{i j}\right|
$$

Specially, for a pure state $|\phi\rangle=\sum a_{i}|i\rangle$, the $l_{1}$ norm of coherence is

$$
C_{l_{1}}(\phi) \equiv \sum_{i \neq j}\left|a_{i} a_{j}\right|
$$

It can also be expressed as

$$
C_{l_{1}}(\phi) \equiv\left(\sum_{i}\left|a_{i}\right|\right)^{2}-1
$$

We consider $l_{1}$ norm of coherence measure and obtain corresponding bounds for coherence of superpositions. See Methods for the proof of the following Theorem.

Theorem 3. Given two orthogonal states $|\Phi\rangle,|\Psi\rangle$, and two complex number $\alpha, \beta$ satisfying $\| \alpha|\Phi\rangle+\beta|\Psi\rangle \|=1$, the coherence of the superposition $|\Omega\rangle=\alpha|\Phi\rangle+\beta|\Psi\rangle$ satisfies

$$
C_{l_{1}}(\Omega) \leq \min \left\{\begin{array}{l}
|\alpha|^{2} C_{l_{1}}(\Phi)+|\beta|^{2} C_{l_{1}}(\Psi)+2|\alpha \beta|(n-1), \\
|\alpha|^{2} C_{l_{1}}(\Phi)+|\beta|^{2} C_{l_{1}}(\Psi)+2|\alpha \beta| \sqrt{\left(C_{l_{1}}(\Phi)+1\right)\left(C_{l_{1}}(\Psi)+1\right)},
\end{array}\right.
$$

where $n$ is the dimension of Hilbert space.

Remark 3: An interesting symmetric inequality can be deduced from the second line in Eq. (14) as follows:

$$
\sqrt{C_{l_{1}}(\Omega)+1} \leq|\alpha| \sqrt{C_{l_{1}}(\Phi)+1}+|\beta| \sqrt{C_{l_{1}}(\Psi)+1} .
$$

This inequality may have applications in other analysis of coherence by considering superposition effects.

Remark 4: Here, we want to emphasize that in Theorem 3 the dimension $n$ is an arbitrary positive integer and our result covers the two dimension case mentioned in ref. 33 .

Now, we compare the values of two expressions in Theorem 3 . The only difference between them locates at the last term. One is $2|\alpha \beta|(n-1)$, and the other is $2|\alpha \beta| \sqrt{\left(C_{l_{1}}(\Phi)+1\right)\left(C_{l_{1}}(\Psi)+1\right)}$. Thus, we only need to compare the values of $n-1$ and $\sqrt{\left(C_{l_{1}}(\Phi)+1\right)\left(C_{l_{1}}(\Psi)+1\right)}$. In Figure 2, we plot function $\sqrt{\left(C_{l_{1}}(\Phi)+1\right)\left(C_{l_{1}}(\Psi)+1\right)}$ by setting $C_{l_{1}}(\Phi)$ and $C_{l_{1}}(\Psi)$ as independent variables whose domains are $[0,10]$. Since $n-1$ is an integer, it is easy for us to compare the value of $\sqrt{\left(C_{l_{1}}(\Phi)+1\right)\left(C_{l_{1}}(\Psi)+1\right)}$ and coordinate axis scale which can be recognized as $n-1$. If $n-1$ is smaller than the value of $\sqrt{\left(C_{l_{1}}(\Phi)+1\right)\left(C_{l_{1}}(\Psi)+1\right)}$, then the upper bound will be $|\alpha|^{2} C_{l_{1}}(\Phi)+|\beta|^{2} C_{l_{1}}(\Psi)+2|\alpha \beta|(n-1)$. If $n-1$ is larger than the value of $\sqrt{\left(C_{l_{1}}(\Phi)+1\right)\left(C_{l_{1}}(\Psi)+1\right)}$, the $|\alpha|^{2} C_{l_{1}}(\Phi)+|\beta|^{2} C_{l_{1}}(\Psi)+2|\alpha \beta| \sqrt{\left(C_{l_{1}}(\Phi)+1\right)\left(C_{l_{1}}(\Psi)+1\right)}$ is the tighter upper bound.

Next, we focus on discussing lower bound. Through using the absolute value inequality, we can obtain the lower bound for $l_{1}$ norm of coherence of superpositions as illustrated in the following theorem. Its proof can be found in Methods. 
Theorem 4. Given two orthogonal states $|\Phi\rangle,|\Psi\rangle$, and two complex number $\alpha, \beta$ satisfying $\| \alpha|\Phi\rangle+\beta|\Psi\rangle \|=1$, the coherence of the superposition $|\Omega\rangle=\alpha|\Phi\rangle+\beta|\Psi\rangle$ satisfies

$$
C_{l_{1}}(\Omega) \geq \max \left\{\begin{array}{l}
|\alpha|^{2} C_{l_{1}}(\Phi)+|\beta|^{2} C_{l_{1}}(\Psi)-2|\alpha \beta|(n-1), \\
|\alpha|^{2} C_{l_{1}}(\Phi)+|\beta|^{2} C_{l_{1}}(\Psi)-2|\alpha \beta| \sqrt{\left(C_{l_{1}}(\Phi)+1\right)\left(C_{l_{1}}(\Psi)+1\right)}, \\
0,
\end{array}\right.
$$

where $n$ is the dimension of Hilbert space.

Remark 5: An interesting symmetric inequality can be deduced from the second line in Eq. (16) as follows:

$$
\sqrt{C_{l_{1}}(\Omega)+1} \geq|| \alpha\left|\sqrt{C_{l_{1}}(\Phi)+1}-\right| \beta\left|\sqrt{C_{l_{1}}(\Psi)+1}\right| .
$$

Same as the Eq. (15), this inequality may have applications in other analysis of coherence by considering superposition effects.

The only difference between the first line and second line in Eq. (16) is also in the last term, which are $-2|\alpha \beta|(n-1)$ and $-2|\alpha \beta| \sqrt{\left(C_{l_{1}}(\Phi)+1\right)\left(C_{l_{1}}(\Psi)+1\right)}$ respectively. This comparison is dimension dependent as shown in Fig. 2. Note that since a measure of coherence is nonnegative, we compare the expressions in Eq. (16) and choose the maximum value as the lower bound.

Robustness of coherence. As a quantifier of the advantage enabled by a quantum state in phase discrimination task, the robustness of coherence is defined and proved to be a full measure for the framework proposed in ref. 13. Robustness of coherence is shown to be an observable related to the notion of coherence witness ${ }^{17,18}$. Given a fixed basis $\left\{|i\rangle_{i=1}^{n}\right\}$, where $n$ is the dimension of Hilbert space. The definition of robustness of coherence of a state $\rho$ is as following ${ }^{17}$ :

$$
C_{R}(\rho)=\min _{\tau \in D\left(\mathbb{C}^{d}\right)}\left\{s \geq 0 \mid \frac{\rho+s \tau}{1+s}=: \delta \in \mathcal{I}\right\},
$$

where $D\left(\mathbb{C}^{d}\right)$ is the convex set of density operators acting on a $n$-dimensional Hilbert space, and $\mathcal{I}$ is the set of all incoherent states. Notice that the robustness of coherence of a pure state $|\phi\rangle$ satisfies ${ }^{18}$,

$$
C_{R}(\phi)=C_{l_{1}}(\phi)
$$

From this equation, we can see the equivalence between robustness of coherence and $l_{1}$ norm of coherence for pure states. The bounds are same as in Theorem 3 and Theorem 4.

Coherence of superpositions for two states from orthogonal subspaces. Quantum states from orthogonal subspaces play an important role in quantum information and coding ${ }^{36}$. Here we consider the bounds for this special case. That is, the decomposition states $(|\Phi\rangle$ and $|\Psi\rangle)$ come from orthogonal subspaces.

Corollary 1. Let $|\Phi\rangle=\sum_{i=1}^{n} a_{i}|i\rangle,|\Psi\rangle=\sum_{i=1}^{n} b_{i}|i\rangle$ be two states satisfying $a_{i} b_{i}=0, i=1, \ldots, n(|\Phi\rangle$ and $|\Psi\rangle$ belong to two orthogonal subspaces), and $|\Omega\rangle=\alpha|\Phi\rangle+\beta|\Psi\rangle,|\alpha|^{2}+|\beta|^{2}=1$. Then the coherence of the superposition state $|\Omega\rangle$ has the following bounds:

(I) for relative entropy of coherence

$$
C_{r e}(\Omega)=|\alpha|^{2} C_{r e}(\Phi)+|\beta|^{2} C_{r e}(\Psi)+h_{2}\left(|\alpha|^{2}\right),
$$

$$
\text { where }|\alpha|^{2}+|\beta|^{2}=1 \text { and } h_{2}(x) \equiv-x \log x-(1-x) \log (1-x) \text {. }
$$

(II) for $l_{1}$ norm of coherence

$$
C_{l_{1}}(\Omega) \leq \min \left\{\begin{array}{l}
|\alpha|^{2} C_{l_{1}}(\Phi)+|\beta|^{2} C_{l_{1}}(\Psi)+n|\alpha \beta|, \\
|\alpha|^{2} C_{l_{1}}(\Phi)+|\beta|^{2} C_{l_{1}}(\Psi)+2|\alpha \beta| \sqrt{\left(C_{l_{1}}(\Phi)+1\right)\left(C_{l_{1}}(\Psi)+1\right)},
\end{array}\right.
$$

and

$$
C_{l_{1}}(\Omega) \geq|\alpha|^{2} C_{l_{1}}(\Phi)+|\beta|^{2} C_{l_{1}}(\Psi)+2|\alpha \beta| .
$$

For the superposition of two states from two orthogonal subspaces, the relative entropy coherence is the sum of three terms: the average of the coherence of two states being superposed, the binary entropy of probability $|\alpha|^{2}$. Instead of bounds, this is an accurate expression as shown in Eq. (20). The maximum increase for coherence is bounded as following: 


\begin{tabular}{|c|c|}
\hline upper bound for partial entropy of entanglement ${ }^{29}$ & upper bound for relative entropy of coherence \\
\hline$\frac{1}{q}\left[p E(\Phi)+(1-p) E(\Psi)+h_{2}(p)\right]$ & $\min \left\{\begin{array}{l}\frac{1}{q} f(p) \\
f(p)+2(1-q)|\alpha||\beta| \log (n-1)+(1-q)\end{array}\right.$ \\
\hline lower bound for partial entropy of entanglement ${ }^{29}$ & lower bound for relative entropy of coherence \\
\hline $\max \left\{\begin{array}{l}\frac{(1-p)|\alpha|^{2}}{1-p|\alpha|^{2}} E(\Phi)-\frac{1-p}{p} E(\Psi)-\frac{1}{p} h_{2}(p) \\
\frac{(1-p)|\beta|^{2}}{1-p|\beta|^{2}} E(\Phi)-\frac{1-p}{p} E(\Psi)-\frac{1}{p} h_{2}(p)\end{array}\right.$ & $\max \left\{\begin{array}{l}\frac{(1-p)|\alpha|^{2}}{1-p|\alpha|^{2}} C_{r e}(\Phi)-\frac{1-p}{p} C_{r e}(\Psi)-\frac{1}{p} h_{2}(p) \\
\frac{(1-p)|\beta|^{2}}{1-p|\beta|^{2}} C_{r e}(\Phi)-\frac{1-p}{p} C_{r e}(\Psi)-\frac{1}{p} h_{2}(p)\end{array}\right.$ \\
\hline
\end{tabular}

Table 1. Comparison between bounds on partial entropy of entanglement and relative entropy coherence for the superposition of two orthogonal states (Here $E\left(\phi^{A B}\right)=S\left(\operatorname{Tr}_{A}|\phi\rangle\langle\phi|\right)$ is an entanglement measure of pure state $\left.\phi^{A B 28}\right)$.

\begin{tabular}{|c|c|}
\hline upper bound for concurrence ${ }^{31}$ & upper bound for $l_{1}$ norm of coherence \\
\hline$|\alpha|^{2} E_{C}(\Phi)+|\beta|^{2} E_{C}(\Psi)+2|\alpha \beta|$ & $\min \left\{\begin{array}{l}|\alpha|^{2} C_{l_{1}}(\Phi)+|\beta|^{2} C_{l_{1}}(\Psi)+2(n-1)|\alpha \beta| \\
|\alpha|^{2} C_{l_{1}}(\Phi)+|\beta|^{2} C_{l_{1}}(\Psi)+2|\alpha \beta| \sqrt{\left(C_{l_{1}}(\Phi)+1\right)\left(C_{l_{1}}(\Psi)+1\right)}\end{array}\right.$ \\
\hline lower bound for concurrence ${ }^{31}$ & lower bound for $l_{1}$ norm of coherence \\
\hline $\begin{array}{l}|\alpha|^{2} E_{C}(\Phi)-|\beta|^{2} E_{C}(\Psi)-2|\alpha \beta| \text { or } \\
|\beta|^{2} E_{C}(\Psi)-|\alpha|^{2} E_{C}(\Phi)-2|\alpha \beta|\end{array}$ & $\max \left\{\begin{array}{l}|\alpha|^{2} C_{l_{1}}(\Phi)+|\beta|^{2} C_{l_{1}}(\Psi)-2(n-1)|\alpha \beta| \\
|\alpha|^{2} C_{l_{1}}(\Phi)+|\beta|^{2} C_{l_{1}}(\Psi)-2|\alpha \beta| \sqrt{\left(C_{l_{1}}(\Phi)+1\right)\left(C_{l_{1}}(\Psi)+1\right)}\end{array}\right.$ \\
\hline
\end{tabular}

Table 2. Comparison between bounds on concurrence and $l_{1}$ norm coherence for the superposition of two orthogonal states (Here $E_{C}$ is concurrence which is a measure of entanglement ${ }^{31,38}$ ).

$$
C_{r e}(\Omega)-|\alpha|^{2} C_{r e}(\Phi)-|\beta|^{2} C_{r e}(\Psi) \leq 1 .
$$

The bounds for $l_{1}$ norm coherence of superposition considering orthogonal subspaces in Corollary 1 is tighter compared with the general case in Theorem 3 and 4. Specifically, the expression given in the first line of Eq. (21) is lowered and the expression given in Eq. (22) is raised.

\section{Discussion}

Using the relative entropy of coherence, $l_{1}$ norm of coherence and robustness of coherence, we give the upper and lower bounds for coherence of the superposition. Bounds for coherence of superpositions of multiple terms can be easily found by generalizing our methods.

Superposition is the root of both coherence and entanglement. Coherence is a property of an arbitrary quantum state, while entanglement is a property of a bipartite or multipartite state. In this sense, it is straightforward to understand that coherence is a more general quantum property than entanglement ${ }^{20}$. Entanglement of the superposition is closely linked to the entanglement of these two superposed states ${ }^{28,29,31}$. In this work, we have shown that the coherence of the superposition is intimately related to the coherence of these two superposed states. However, it is still unclear that the difference between entanglement of superpositions and coherence of superpositions. As shown in Tables 1 and 2, there exist strong similarities between coherence and entanglement for the analogy problem.

From the expressions in Table 2, we can see that the upper bound and lower bound for coherence are symmetric about the statistical average coherence of the two superposed orthogonal states, which is defined as $|\alpha|^{2} C_{l_{1}}(\Phi)+|\beta|^{2} C_{l_{1}}(\Psi)$, and the green line as shown in Fig. 2(a). While the bounds for entanglement are asymmetric around the statistical average coherence of the two superposed orthogonal states defined as $|\alpha|^{2} E_{C}(\Phi)+|\beta|^{2} E_{C}(\Psi)$, and the green line as shown in Fig. 2(b). Here, we consider the case where our lower bound is valid, i.e. positive. In the following, we give an intuitive comparison between coherence and entanglement for Table 2 by taking a simple example. We focus on fluctuating ranges of $l_{1}$ norm coherence and concurrence for the superposition of two orthogonal states. Here in order to make the discussion meaningful we consider the superposition of two bipartite orthogonal states.

Example 2: Let $|\Psi\rangle$ and $|\Phi\rangle$ be two orthogonal states, defined by

$$
|\Psi\rangle=\frac{|00\rangle+|11\rangle}{\sqrt{2}} \text { and }|\Phi\rangle=\frac{|00\rangle+\sqrt{2}|01\rangle+|10\rangle-|11\rangle}{\sqrt{5}} .
$$

Their coherence and entanglement are depicted in Fig. 3. Evidently, the fluctuation of coherence is smoother than entanglement. The trend of change for coherence and entanglement with respect to the parameter $|\alpha|^{2}$ is not positive correlated with each other. Furthermore, there are two inflections points in the change of exact value of coherence while only one for entanglement considering the change of $|\alpha|^{2}$ in the case of example 2. Also in this 

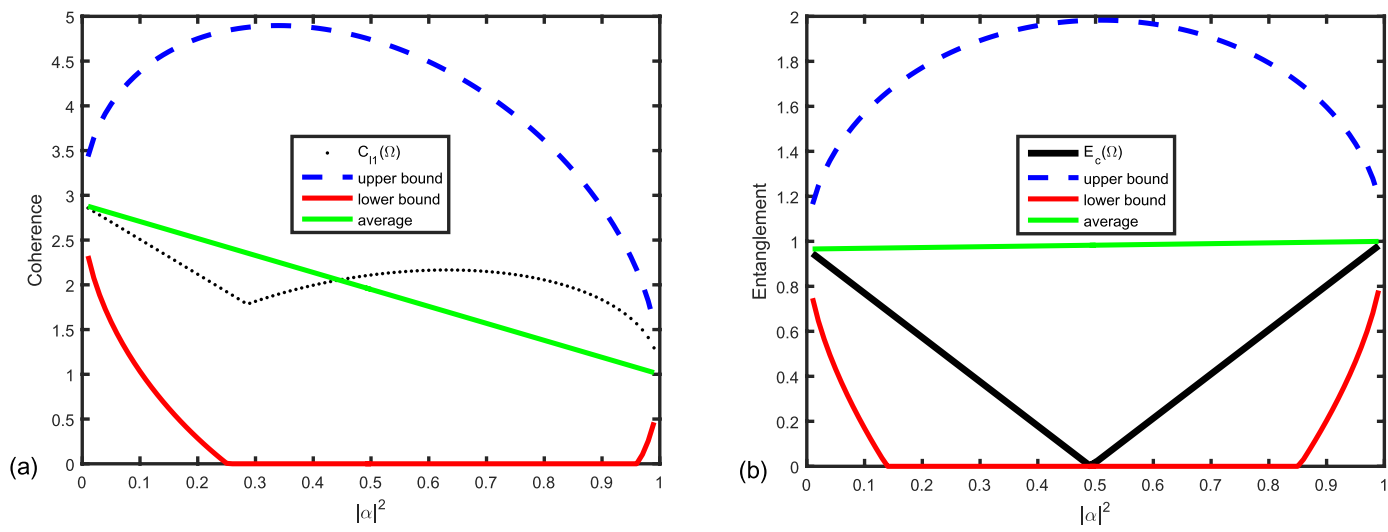

Figure 3. Bounds for $l_{1}$ coherence (a) and concurrence (b) of the two states in Eq. (24). In sub-figure (a), the black dotted line is the actual values of $l_{1}$ coherence, the blue dashed line is upper bound on $l_{1}$ coherence, the red solid line is lower bound on $l_{1}$ coherence, and the green line represents the statistical average coherence of the superposed states, $|\alpha|^{2} C_{l_{1}}(\Phi)+|\beta|^{2} C_{l_{1}}(\Psi)$. In sub-figure (b), the black dotted line is the actual values of concurrence, the blue dashed line is upper bound on concurrence, the red solid line is lower bound on concurrence, and the green line represents the statistical average entanglement of the superposed states, $|\alpha|^{2} E_{C}(\Phi)+|\beta|^{2} E_{C}(\Psi)$. Note that coherence and entanglement are both non-negative.

example we can see that when entanglement of the superposition state disappears, the coherence still exists. From these observations, we can see that coherence is a kind of resource easier accessed compared with entanglement.

In some information processing tasks, such as coherence distillation, we are more concerned with the coherence of the state, rather than the state itself. When we only know the coherence value of $|\Phi\rangle$ and $|\Psi\rangle$, and do not know what the specific state is, we can use our results to estimate the upper and lower bound of the superposition, then we can know that the least amount and the most amount of coherence we can distill from the superposition state.

Another interesting and challenging problem related to our work is the effects of superposition on the coherence of superposition of mixed states, which can be used to analyze the effects of de-coherence resulted from the interactions with environment.

\section{Methods}

Proof of the Theorem 1. We construct the state $|\chi\rangle^{S A}=\sqrt{p}|\Phi\rangle^{S}|0\rangle^{A}+\sqrt{1-p}|\Psi\rangle^{S}|1\rangle^{A}$, where we used an ancillary system $A$. The coherence of $|\chi\rangle$ can be expressed as $p C_{r e}(\Phi)+(1-p) C_{r e}(\Psi)+h_{2}(p)$. Through measuring ancillary system $A$ with Kraus operators $\left\{|0\rangle\left(\cos \theta e^{i \omega_{1}}\left\langle 0\left|+\sin \theta e^{i \omega_{2}}\langle 1|\right), \mid 1\right\rangle\left(-\sin \theta e^{-i \omega_{2}}\langle 0|+\cos \theta e^{-i \omega_{1}}\langle 1|\right)\right\}\right.$, with probability $\left.q=\| \sqrt{p} \cos \theta e^{i \omega_{1}}|\Phi\rangle+\sqrt{1-p} \sin \theta e^{i \omega_{2}}|\Psi\rangle\right) \|^{2}, \quad$ the state becomes $|\Omega\rangle|0\rangle=\left(\sqrt{\frac{p}{q}} \cos \theta e^{i \omega_{1}}|\Phi\rangle+\sqrt{\frac{1-p}{q}} \sin \theta e^{i \omega_{2}}|\Psi\rangle\right)|0\rangle$ and with probability $1-q$, the state becomes $|\Gamma\rangle|1\rangle=\left(-\sqrt{\frac{p}{1-q}} \sin \theta e^{-i \omega_{2}}|\Phi\rangle+\sqrt{\frac{1-p}{1-q}} \cos \theta e^{-i \omega_{1}}|\Psi\rangle\right)|1\rangle$. This measurement is an incoherent operation and $C_{r e}$ is a coherence monotone ${ }^{13}$, we can get the following inequality

$$
q C_{r e}(\Omega)+(1-q) C_{r e}(\Gamma) \leq p C_{r e}(\Phi)+(1-p) C_{r e}(\Psi)+h_{2}(p) .
$$

Since $C_{r e}(\Gamma) \geq 0$, we can get the following inequality

$$
C_{r e}(\Omega) \leq \frac{1}{q}\left[p C_{r e}(\Phi)+(1-p) C_{r e}(\Psi)+h_{2}(p)\right]=\frac{1}{q} f(p)
$$

(1) Now, we proof the first line in Eq. (7) using the similar method in ref. 29 first. Here we set

$$
\frac{\alpha}{\| \alpha|\Phi\rangle+\beta|\Psi\rangle \|}=\sqrt{\frac{p}{q}} \cos \theta e^{i \omega_{1}} \text { and } \frac{\beta}{\| \alpha|\Phi\rangle+\beta|\Psi\rangle \|}=\sqrt{\frac{1-p}{q}} \sin \theta e^{i \omega_{2}} .
$$

It is straightforward to get

$$
|\alpha|^{2}=\frac{p}{q} \cos ^{2} \theta \| \alpha|\Phi\rangle+\beta|\Psi\rangle \|^{2} \text { and }|\beta|^{2}=\frac{1-p}{q} \sin ^{2} \theta \| \alpha|\Phi\rangle+\beta|\Psi\rangle \|^{2} .
$$

Due to $|\alpha|^{2}+|\beta|^{2}=1$, and we can obtain 


$$
\cos ^{2} \theta=\frac{(1-p)|\alpha|^{2}}{(1-p)|\alpha|^{2}+p|\beta|^{2}}
$$

Through substituting Eq. (29) into Eq. (28), and we can obtain

$$
q=\frac{p(1-p)}{(1-p)|\alpha|^{2}+p|\beta|^{2}} \| \alpha|\Phi\rangle+\beta|\Psi\rangle \|^{2} .
$$

Finally, substitute Eq. (30) into Eq. (26), and the result can be proved

$$
\begin{aligned}
C_{r e}(\Omega) & \leq \frac{(1-p)|\alpha|^{2}+p|\beta|^{2}}{p(1-p) \| \alpha|\Phi\rangle+\beta|\Psi\rangle \|^{2}}\left[p C_{r e}(\Phi)+(1-p) C_{r e}(\Psi)+h_{2}(p)\right] \\
& =\frac{1}{q} f(p),
\end{aligned}
$$

with $0<p<1$.

(2) The other bound in Eq. (7) is obtained through getting a tighter lower bound of $C_{r e}(\Gamma)$. Firstly, we introduce two lemmas on which the proof is based.

Lemma 1. (Fannes-Audenaert inequality ${ }^{37}$ ) Suppose $\rho$ and $\sigma$ are density matrices such that the trace distance is given by $T$,

$$
|S(\rho)-S(\sigma)| \leq T \log (d-1)+h_{2}(T),
$$

where $d$ is the dimension of the Hilbert space, and $h_{2}(x)=-x \log x-(1-x) \log (1-x)$.

Lemma 2. Given two orthogonal states $|\Phi\rangle,|\Psi\rangle$, and two complex number $\alpha, \beta$ satisfying $\| \alpha|\Phi\rangle+\beta|\Psi\rangle \|=1$. Let $|\Omega\rangle=\alpha|\Phi\rangle+\beta|\Psi\rangle,|\Gamma\rangle=\alpha|\Phi\rangle-\beta|\Psi\rangle$, and $|\Omega\rangle_{d}\langle\Omega|,| \Gamma\rangle_{d}\langle\Gamma|$ are the diagonal matrices of $|\Omega\rangle\langle\Omega|,| \Gamma\rangle\langle\Gamma|$ respectively. The trace distance between $|\Omega\rangle_{d}\langle\Omega|$ and $|\Gamma\rangle_{d}\langle\Gamma|$ satisfies

$$
T\left(|\Omega\rangle_{d}\langle\Omega|,| \Gamma\rangle_{d}\langle\Gamma|\right) \leq 2|\alpha \beta| .
$$

Proof: Given $|\Phi\rangle=\sum_{i=0}^{n-1} a_{i}|i\rangle$, and $|\Psi\rangle=\sum_{i=0}^{n-1} b_{i}|i\rangle$,

$$
\begin{aligned}
& |\Omega\rangle=\alpha|\Phi\rangle+\beta|\Psi\rangle=\sum_{i=0}^{n-1}\left(\alpha a_{i}+\beta b_{i}\right)|i\rangle, \\
& |\Gamma\rangle=\alpha|\Phi\rangle-\beta|\Psi\rangle=\sum_{i=0}^{n-1}\left(\alpha a_{i}-\beta b_{i}\right)|i\rangle .
\end{aligned}
$$

Then we can get the diagonal matrices of $|\Omega\rangle\langle\Omega|$ and $|\Gamma\rangle\langle\Gamma|$ by deleting all off-diagonal elements as follow

$$
\begin{aligned}
|\Omega\rangle_{d}\langle\Omega| & =\sum\left(\alpha a_{i}+\beta b_{i}\right)\left(\alpha^{*} a_{i}^{*}+\beta^{*} b_{i}^{*}\right)|i\rangle\langle i|, \\
|\Gamma\rangle_{d}\langle\Gamma| & =\sum\left(\alpha a_{i}-\beta b_{i}\right)\left(\alpha^{*} a_{i}^{*}-\beta^{*} b_{i}^{*}\right)|i\rangle\langle i| .
\end{aligned}
$$

The trace distance between $|\Omega\rangle_{d}\langle\Omega|$ and $|\Gamma\rangle_{d}\langle\Gamma|$ is

$$
T\left(|\Omega\rangle_{d}\langle\Omega|,| \Gamma\rangle_{d}\langle\Gamma|\right)=\frac{1}{2} \operatorname{tr}|R| .
$$

Here, we denote $R=|\Omega\rangle_{d}\langle\Omega|-| \Gamma\rangle_{d}\langle\Gamma|$ for short.

$$
\begin{aligned}
|R| & =\sqrt{R^{+} R} \\
& =\sum \sqrt{4\left(\alpha^{*} \beta a_{i}^{*} b_{i}+\alpha \beta^{*} a_{i} b_{i}^{*}\right)^{2}}|i\rangle\langle i| \\
& =\sum \sqrt{16|\alpha|^{2}|\beta|^{2}\left|a_{i}\right|^{2}\left|b_{i}\right|^{2}}|i\rangle\langle i| \\
& =\sum 4|\alpha||\beta|\left|a_{i}\right|\left|b_{i}\right||i\rangle\langle i| .
\end{aligned}
$$

The upper bound of trace distance between $|\Omega\rangle_{d}\langle\Omega|$ and $|\Gamma\rangle_{d}\langle\Gamma|$ can be get as follows: 


$$
\begin{aligned}
T\left(|\Omega\rangle_{d}\langle\Omega|,| \Gamma\rangle_{d}\langle\Gamma|\right) & =\frac{1}{2} \operatorname{tr}|R| \\
& =2|\alpha||\beta| \sum\left|a_{i}\right|\left|b_{i}\right| \\
& \leq 2|\alpha||\beta| \sum\left(\frac{\left|a_{i}\right|^{2}+\left|b_{i}\right|^{2}}{2}\right) \\
& =2|\alpha||\beta|,
\end{aligned}
$$

where the first inequality is due to fundamental inequality, and the last equality is due to the $\sum_{i}\left|a_{i}\right|^{2}=\sum_{i}\left|b_{i}\right|^{2}=1$.

We can get the following inequality from lemma 1 and 2

$$
\begin{aligned}
\left|C_{r e}(\Omega)-C_{r e}(\Gamma)\right| & \leq T\left(|\Omega\rangle_{d}\langle\Omega|,| \Gamma\rangle_{d}\langle\Gamma|\right) \log (n-1)+h_{2}\left(T\left(|\Omega\rangle_{d}\langle\Omega|,| \Gamma\rangle_{d}\langle\Gamma|\right)\right) \\
& \leq 2|\alpha||\beta| \log (n-1)+1 .
\end{aligned}
$$

Furthermore, we can get

$$
(1-q) C_{r e}(\Omega)-(1-q) C_{r e}(\Gamma) \leq 2(1-q)|\alpha||\beta| \log (n-1)+(1-q),
$$

and through shifting one term in LHS we can get

$$
(1-q) C_{r e}(\Gamma) \geq(1-q) C_{r e}(\Omega)-2(1-q)|\alpha||\beta| \log (n-1)-(1-q) .
$$

Substitute Eq. (42) into Eq. (25), then we can get

$$
\begin{aligned}
& q C_{r e}(\Omega)+(1-q) C_{r e}(\Omega)-2(1-q)|\alpha||\beta| \log (n-1)-(1-q) \\
& \quad \leq q C_{r e}(\Omega)+(1-q) C_{r e}(\Gamma) \leq f(p) .
\end{aligned}
$$

After simplification, we can get the result. Note that this upper bound is dimension dependent.

Proof of the Theorem 2. Let $|\Omega\rangle=\alpha|\Phi\rangle+\beta|\Psi\rangle$. Through measuring the state $\left|\chi^{\prime}\right\rangle^{S A}=\sqrt{p}|\Omega\rangle^{S}|0\rangle^{A}+\sqrt{1-p}|\Psi\rangle^{S}|1\rangle^{A}$, where we used an ancillary system A. The coherence of $\left|\chi^{\prime}\right\rangle$ can be expressed as $p C_{r e}(\Omega)+(1-p) C_{r e}(\Psi)+h_{2}(p)$. We measure ancillary system A with Kraus operators $\left\{|0\rangle\left(\cos \theta e^{i \omega_{1}}\left\langle 0\left|+\sin \theta e^{i \omega_{2}}\langle 1|\right), \mid 1\right\rangle\left(-\sin \theta e^{-i \omega_{2}}\langle 0|+\cos \theta e^{-i \omega_{1}}\langle 1|\right)\right\}\right.$, where $\beta=|\beta| e^{i\left(\omega_{2}-\omega_{1}\right)}$. With probability $t=\| \sqrt{p} \cos \theta e^{i \omega_{1}}|\Omega\rangle+\sqrt{1-p} \sin \theta e^{i \omega_{2}}|\Psi\rangle \|^{2} \quad$ t h e $\quad$ s $\quad \mathrm{t}$ a $\mathrm{t}$ e $\quad$ b e c c o m e $\mathrm{s}$ $\left|\Gamma_{1}\right\rangle|0\rangle=\left(\sqrt{\frac{p}{t}} \cos \theta e^{i \omega_{1}}|\Omega\rangle+\sqrt{\frac{1-p}{t}} \sin \theta e^{i \omega_{2}}|\Psi\rangle\right)|0\rangle$ and with probability $1-t$ the state becomes $\left|\Gamma_{2}\right\rangle|1\rangle=\left(-\sqrt{\frac{p}{1-t}} \sin \theta e^{-i \omega_{2}}|\Omega\rangle+\sqrt{\frac{1-p}{1-t}} \cos \theta e^{-i \omega_{1}}|\Psi\rangle\right)|1\rangle$. This measurement is an incoherent operation and $C_{r e}$ is a coherence monotone ${ }^{13}$, we can get

$$
t C_{r e}\left(\Gamma_{1}\right)+(1-t) C_{r e}\left(\Gamma_{2}\right) \leq p C_{r e}(\Omega)+(1-p) C_{r e}(\Psi)+h_{2}(p) .
$$

Since $C_{r e}\left(\Gamma_{2}\right) \geq 0$, we can obtain

$$
C_{r e}(\Omega) \geq \frac{1}{p}\left[t C_{r e}\left(\Gamma_{1}\right)-(1-p) C_{r e}(\Psi)-h_{2}(p)\right] .
$$

Through the following setting like in ref. 29, we can obtain $\left|\Gamma_{1}\right\rangle=|\Phi\rangle$

$$
\begin{gathered}
\alpha \sqrt{\frac{p}{t}} \cos \theta e^{i \omega_{1}}=1, \\
\beta \sqrt{\frac{p}{t}} \cos \theta e^{i \omega_{1}}+\sqrt{\frac{1-p}{t}} \sin \theta e^{i \omega_{2}}=0 .
\end{gathered}
$$

The parameters $\alpha$ and $\beta$ satisfying Eqs (46) and (47) are as follows

$$
\alpha=\sqrt{\frac{t}{p}} \frac{e^{-i \omega_{1}}}{\cos \theta}, \text { and } \beta=-\sqrt{\frac{1-p}{p}} \frac{\sin \theta e^{i \omega_{2}}}{\cos \theta e^{i \omega_{1}}} .
$$

and

$$
|\alpha|^{2}=\frac{t}{p \cos ^{2} \theta} \text { and }|\beta|^{2}=\frac{(1-p) \sin ^{2} \theta}{p \cos ^{2} \theta}=\frac{|\alpha|^{2}(1-p) \sin ^{2} \theta}{t} .
$$

We can obtain 


$$
t=\frac{p(1-p)|\alpha|^{2}}{1-p|\alpha|^{2}}
$$

Substitute Eq. (50) into Eq. (45), then the results in Theorem 2 can be proved

$$
C_{r e}(\Omega) \geq \frac{(1-p)|\alpha|^{2}}{1-p|\alpha|^{2}} C_{r e}(\Phi)-\frac{1-p}{p} C_{r e}(\Psi)-\frac{1}{p} h_{2}(p),
$$

with $0<p<1$. As such, the another lower bound can be obtained by exchanging $|\Phi\rangle$ and $|\Psi\rangle$.

Proof of the Theorem 3. Given two $n$ dimensional states $|\Phi\rangle=\sum_{i}^{n} a_{i}|i\rangle$ and $|\Psi\rangle=\sum_{i}^{n} b_{i}|i\rangle$, where $a_{i}, b_{i}$ are complex numbers and satisfied $\sum\left|a_{i}\right|^{2}=\sum\left|b_{i}\right|^{2}=1$. Let $|\Omega\rangle=\alpha|\Phi\rangle+\beta|\Psi\rangle$, and $\|\Omega\|=1$.

(1) Firstly, we prove the first upper bound in Eq. (14). From the definition of $l_{1}$ norm of coherence Eq. (12). We have

$$
\begin{aligned}
& C_{l_{1}}(\Phi)=\sum_{i \neq j}\left|a_{i} a_{j}\right|, \\
& C_{l_{1}}(\Psi)=\sum_{i \neq j}\left|b_{i} b_{j}\right| .
\end{aligned}
$$

and

$$
\begin{aligned}
C_{l_{1}}(\Omega) & =\sum_{i \neq j}\left|\left(\alpha a_{i}+\beta b_{i}\right)\left(\alpha a_{j}+\beta b_{j}\right)\right| \\
& \leq \sum_{i \neq j}\left(|\alpha|^{2}\left|a_{i} a_{j}\right|+|\beta|^{2}\left|b_{i} b_{j}\right|+2|\alpha \beta|\left|a_{i} b_{j}\right|\right) \\
& =|\alpha|^{2} \sum_{i \neq j}\left|a_{i} a_{j}\right|+|\beta|^{2} \sum_{i \neq j}\left|b_{i} b_{j}\right|+2|\alpha \beta| \sum_{i \neq j}\left|a_{i} b_{j}\right| \\
& =|\alpha|^{2} C_{l_{1}}(\Phi)+|\beta|^{2} C_{l_{1}}(\Psi)+|\alpha \beta| \sum_{i \neq j} 2\left|a_{i} b_{j}\right|,
\end{aligned}
$$

where the first inequality is due to absolute value inequality. Successive application of the mean inequality, we will get

$$
2 \sum_{i \neq j}\left|a_{i} b_{j}\right| \leq(n-1)\left(\sum_{i}\left|a_{i}\right|^{2}+\sum_{j}\left|b_{j}\right|^{2}\right) \leq 2(n-1),
$$

which is the first line in Eq. (14).

(2) Now, we prove the other upper bound in Eq. (14). From the definition of $l_{1}$ norm of coherence Eq. (13), we have

$$
\begin{aligned}
& C_{l_{1}}(\Phi)=\left(\sum_{i}\left|a_{i}\right|\right)^{2}-1, \\
& C_{l_{1}}(\Psi)=\left(\sum_{i}\left|b_{i}\right|\right)^{2}-1 .
\end{aligned}
$$

Hence, we can obtain

$$
\begin{aligned}
C_{l_{1}}(\Omega) & =\left(\sum_{i}\left|\alpha a_{i}+\beta b_{i}\right|\right)^{2}-1 \\
& \leq\left(\sum_{i}\left|\alpha a_{i}\right|+\sum_{i}\left|\beta b_{i}\right|\right)^{2}-1 \\
& =|\alpha|^{2}\left(\sum_{i}\left|a_{i}\right|\right)^{2}+|\beta|^{2}\left(\sum_{i}\left|b_{i}\right|\right)^{2}+2|\alpha \beta|\left|\left(\sum_{i}\left|a_{i}\right|\right)\left(\sum_{i}\left|b_{i}\right|\right)\right|-1 \\
& =|\alpha|^{2} C_{l_{1}}(\Phi)+|\beta|^{2} C_{l_{1}}(\Psi)+2|\alpha \beta|\left|\left(\sum_{i}\left|a_{i}\right|\right)\left(\left|\sum_{i}\right| b_{i} \mid\right)\right|
\end{aligned}
$$

where the first inequality is due to absolute value inequality. Further, we have 


$$
\left|\left(\sum_{i}\left|a_{i}\right|\right)\left(\left|\sum_{i}\right| b_{i} \mid\right)\right|=\sqrt{\left(\sum_{i}\left|a_{i}\right|\right)^{2}\left(\sum_{i}\left|b_{i}\right|\right)^{2}}=\sqrt{\left(C_{l_{1}}(\Phi)+1\right)\left(C_{l_{1}}(\Psi)+1\right)} .
$$

The upper bound can be gained by substituting Eq. (57) into Eq. (56).

Proof of the Theorem 4. Given two $n$ dimensional states $|\Phi\rangle=\sum_{i=1}^{n} a_{i}|i\rangle$, and $|\Psi\rangle=\sum_{i=1}^{n} b_{i}|i\rangle$, where $a_{i}$, $b_{i}$, are complex numbers and satisfy $\sum\left|a_{i}\right|^{2}=\sum\left|b_{i}\right|^{2}=1$. Let $|\Omega\rangle=\alpha|\Phi\rangle+\beta|\Psi\rangle$, and $\|\Omega\|=1$.

(1) From the definition of $l_{1}$ norm of coherence Eq. (12), we have

$$
\begin{aligned}
& C_{l_{1}}(\Phi)=\sum_{i \neq j}\left|a_{i} a_{j}\right|, \\
& C_{l_{1}}(\Psi)=\sum_{i \neq j}\left|b_{i} b_{j}\right| .
\end{aligned}
$$

Thus, it has

$$
\begin{aligned}
C_{l_{1}}(\Omega) & =\sum_{i \neq j}\left|\alpha a_{i}+\beta b_{i}\right|\left|\alpha a_{j}+\beta b_{j}\right| \\
& \geq \sum_{i \neq j}\left(\left|\alpha a_{i}\right|-\left|\beta b_{i}\right|\right)\left(\left|\alpha a_{j}\right|-\left|\beta b_{j}\right|\right) \\
& =\sum_{i \neq j}\left(|\alpha|^{2}\left|a_{i} a_{j}\right|+|\beta|^{2}\left|b_{i} b_{j}\right|-2|\alpha \beta|\left|a_{i} b_{j}\right|\right) \\
& =|\alpha|^{2} C_{l_{1}}(\Phi)+|\beta|^{2} C_{l_{1}}(\Psi)-|\alpha \beta| \sum_{i \neq j} 2\left|a_{i} b_{j}\right|,
\end{aligned}
$$

where the first inequality is due to absolute value inequality. Further, we find

$$
-2 \sum_{i \neq j}\left|a_{i} b_{j}\right| \geq-(n-1)\left(\sum_{i}\left|a_{i}\right|^{2}+\sum_{j}\left|b_{j}\right|^{2}\right) \geq-2(n-1) .
$$

Therefore, the first lower bound in Theorem 4 can be obtained

(2) From the definition of $l_{1}$ norm of coherence Eq. (13), we have

$$
\begin{aligned}
& C_{l_{1}}(\Phi)=\left(\sum_{i}\left|a_{i}\right|\right)^{2}-1 \\
& C_{l_{1}}(\Psi)=\left(\sum_{i}\left|b_{i}\right|\right)^{2}-1 .
\end{aligned}
$$

Hence, we can obtain

$$
\begin{aligned}
C_{l_{1}}(\Omega) & =\left(\sum_{i}\left|\alpha a_{i}+\beta b_{i}\right|\right)^{2}-1 \\
& \geq\left(\sum_{i}\left|\alpha a_{i}\right|-\sum_{i}\left|\beta b_{i}\right|\right)^{2}-1 \\
& =|\alpha|^{2}\left(\sum_{i}\left|a_{i}\right|\right)^{2}+|\beta|^{2}\left(\sum_{i}\left|b_{i}\right|\right)^{2}-2|\alpha \beta|\left|\left(\sum_{i}\left|a_{i}\right|\right)\left(\sum_{i}\left|b_{i}\right|\right)\right|-1 \\
& =|\alpha|^{2} C_{l_{1}}(\Phi)+|\beta|^{2} C_{l_{1}}(\Psi)-2|\alpha \beta|\left(\sum_{i}\left|a_{i}\right|\right)\left(\sum_{i}\left|b_{i}\right|\right)
\end{aligned}
$$

The second lower bound in Eq. (14) can be obtained by substituting the following Eq. into Eq. (62)

$$
\left(\sum_{i}\left|a_{i}\right|\right)\left(\sum_{i}\left|b_{i}\right|\right)=\sqrt{\left(\sum_{i}\left|a_{i}\right|\right)^{2}\left(\sum_{i}\left|b_{i}\right|\right)^{2}}=\sqrt{\left(C_{l_{1}}(\Phi)+1\right)\left(C_{l_{1}}(\Psi)+1\right)} .
$$

Proof of the Corollary 1. Given $|\Phi\rangle=\sum_{i=1}^{n} a_{i}|i\rangle,|\Psi\rangle=\sum_{i=1}^{n} b_{i}|i\rangle$ be two states satisfying $a_{i} b_{i}=0$, and $|\Omega\rangle=\alpha|\Phi\rangle+\beta|\Psi\rangle$, and $|\alpha|^{2}+|\beta|^{2}=1$. 
(1) According to the conditions of Corollary 1, we can easily get

$$
|\alpha|^{2} S\left(|\Phi\rangle_{d}\langle\Phi|\right)+|\beta|^{2} S\left(|\Psi\rangle_{d}\langle\Psi|\right)+h\left(|\alpha|^{2}\right)=S\left(|\Omega\rangle_{d}\langle\Omega|\right)
$$

Thus, we can get Eq. (20) in terms of Eq. (6).

(2) Let $m_{1}$ and $m_{2}$ are the number of nonzero probability amplitude of $|\Phi\rangle$ and $|\Psi\rangle$ respectively, and $m_{1}+m_{2} \leq n$. From the definition of $l_{1}$ norm of coherence Eq. (12), we have

$$
\begin{aligned}
C_{l_{1}}(\Omega) & =\sum_{i \neq j}\left|\left(\alpha a_{i}+\beta b_{i}\right)\left(\alpha a_{j}+\beta b_{j}\right)\right| \\
& \leq \sum_{i \neq j}\left(|\alpha|^{2}\left|a_{i} a_{j}\right|+|\beta|^{2}\left|b_{i} b_{j}\right|+2|\alpha \beta|\left|a_{i} b_{j}\right|\right) \\
& =|\alpha|^{2} \sum_{i \neq j}\left|a_{i} a_{j}\right|+|\beta|^{2} \sum_{i \neq j}\left|b_{i} b_{j}\right|+2|\alpha \beta| \sum_{i \neq j}\left|a_{i} b_{j}\right| \\
& =|\alpha|^{2} C_{l_{1}}(\Phi)+|\beta|^{2} C_{l_{1}}(\Psi)+|\alpha \beta| \sum_{i \neq j} 2\left|a_{i} b_{j}\right|,
\end{aligned}
$$

where the first inequality is due to absolute value inequality. Successive application of the mean inequality, we will get

$$
2 \sum_{i \neq j}\left|a_{i} b_{j}\right| \leq m_{2}\left(\sum_{i}\left|a_{i}\right|^{2}\right)+m_{1}\left(\sum_{j}\left|b_{j}\right|^{2}\right) \leq n,
$$

We will get the first upper bound by substituting Eq. (66) into Eq. (65).

Since the second upper bound Eq. (14) presented in Theorem 3 also works for this case, we can get the upper bound for the coherence of superposition of two states from two orthogonal subspaces as shown in Eq. (21).

In the following we give the lower bound for the coherence of superposition of two states from two orthogonal subspaces

$$
\begin{aligned}
C_{l_{1}}(\Omega) & =\sum_{i \neq j}\left|\alpha a_{i}+\beta b_{i}\right|\left|\alpha a_{j}+\beta b_{j}\right| \\
& \geq \sum_{i \neq j}\left(\left|\alpha a_{i}\right|-\left|\beta b_{i}\right|\right)\left(\left|\alpha a_{j}\right|-\left|\beta b_{j}\right|\right) \\
& =\sum_{i \neq j}\left(|\alpha|^{2}\left|a_{i} a_{j}\right|+|\beta|^{2}\left|b_{i} b_{j}\right|-2|\alpha \beta|\left|a_{i} b_{j}\right|\right) \\
& =|\alpha|^{2} C_{l_{1}}(\Phi)+|\beta|^{2} C_{l_{1}}(\Psi)-|\alpha \beta| \sum_{i \neq j} 2\left|a_{i} b_{j}\right| .
\end{aligned}
$$

where the first inequality is due to absolute value inequality. Substitute Eq. (66) into Eq. (67), then we can get

$$
C_{l_{1}}(\Omega) \geq|\alpha|^{2} C_{l_{1}}(\Phi)+|\beta|^{2} C_{l_{1}}(\Psi)-n|\alpha \beta| .
$$

Another way to get the lower bound for the coherence of the superposition of two states from two orthogonal subspaces are as follows:

$$
\begin{aligned}
C_{l_{1}}(\Omega) & =\sum_{i, j}\left|\left(\alpha a_{i}+\beta b_{i}\right)\left(\alpha a_{j}+\beta b_{j}\right)-1\right| \\
& =\sum_{i, j}\left(|\alpha|^{2}\left|a_{i} a_{j}\right|+|\beta|^{2}\left|b_{i} b_{j}\right|+2|\alpha \beta|\left|a_{i} b_{j}\right|-1\right) \\
& =|\alpha|^{2} C_{l_{1}}(\Phi)+|\beta|^{2} C_{l_{1}}(\Psi)+2|\alpha \beta| \sum \sum\left|a_{i} b_{j}\right| \\
& \geq|\alpha|^{2} C_{l_{1}}(\Phi)+|\beta|^{2} C_{l_{1}}(\Psi)+2|\alpha \beta| .
\end{aligned}
$$

Since the lower bound Eq. (16) presented in Theorem 4 also works for this case, we can get the lower bound for the coherence of superposition of two states from two orthogonal subspaces as follows:

$$
\begin{gathered}
|\alpha|^{2} C_{l_{1}}(\Phi)+|\beta|^{2} C_{l_{1}}(\Psi)-n|\alpha \beta|, \\
C_{l_{1}}(\Omega) \geq \max \left\{|\alpha|^{2} C_{l_{1}}(\Phi)+|\beta|^{2} C_{l_{1}}(\Psi)-2|\alpha \beta| \sqrt{\left(C_{l_{1}}(\Phi)+1\right)\left(C_{l_{1}}(\Psi)+1\right)},\right. \\
|\alpha|^{2} C_{l_{1}}(\Phi)+|\beta|^{2} C_{l_{1}}(\Psi)+2|\alpha \beta|, \\
=|\alpha|^{2} C_{l_{1}}(\Phi)+|\beta|^{2} C_{l_{1}}(\Psi)+2|\alpha \beta| .
\end{gathered}
$$




\section{References}

1. Jeong, H., Kim, M. S. \& Lee, J. Quantum-information processing for a coherent superposition state via a mixedentangled coherent channel. Phys. Rev. A 64, 052308 (2001).

2. Streltsov, A. et al. Entanglement and coherence in quantum state merging. Phys. Rev. Lett. 116, 240405 (2016).

3. Nielsen, M. A. \& Chuang, I. L. Quantum computation and quantum information (Cambridge University Press, Cambridge, 2000).

4. Deutsch, D. \& Jozsa, R. Rapid solution of problems by quantum computation. Proc. R. Soc. A 439, 553 (1992).

5. Shor, P. W. Algorithms for quantum computation: Discrete logarithms and factoring. In 35th Annual Symposium on Foundations of Computer Science 124 (1994).

6. Giovannetti, V., Lloyd, S. \& Maccone, L. Quantum-enhanced measurements: beating the standard quantum limit. Science 306, 1330 (2004).

7. Giovannetti, V., Lloyd, S. \& Maccone, L. Advances in quantum metrology. Nature Photon. 5, 222 (2011).

8. Åberg, J. Catalytic coherence. Phys. Rev. Lett. 113, 150402 (2014).

9. Narasimhachar, V. \& Gour, G. Low-temperature thermodynamics with quantum coherence. Nat. Commun. 6 (2015).

10. Lostaglio, M., Korzekwa, K., Jennings, D. \& Rudolph, T. Quantum coherence, time-translation symmetry, and thermodynamics. Phys. Rev. X. 5, 021001 (2015).

11. Rebentrost, P., Mohseni, M. \& Aspuru-Guzik, A. Role of quantum coherence and environmental fluctuations in chromophoric energy transport. J. Phys. Chem. B 113, 9942 (2009).

12. Lloyd, S. Quantum coherence in biological systems. J. Phys. Conf. Ser. 302, 012037 (2011).

13. Baumgratz, T., Cramer, M. \& Plenio, M. B. Quantifying coherence. Phys. Rev. Lett. 113, 140401 (2014).

14. Streltsov, A., Singh, U., Dhar, H. S., Bera, M. N. \& Adesso, G. Measuring Quantum Coherence with Entanglement. Phys. Rev. Lett. 115, 020403 (2015)

15. Yuan, X., Zhou, H., Cao, Z. \& Ma, X. Intrinsic randomness as a measure of quantum coherence. Phys. Rev. A 92, 022124 (2015).

16. Rana, S., Parashar, P. \& Lewenstein, M. Trace-distance measure of coherence. Phys. Rev. A 93, 012110 (2016).

17. Napoli, C. et al. Robustness of coherence: An operational and observable measure of quantum coherence. Phys. Rev. Lett. 116, $150502(2016)$.

18. Piani, M. et al. Robustness of asymmetry and coherence of quantum states. Phys. Rev. A 93, 042107 (2016).

19. Yao, Y., Xiao, X., Ge, L. \& Sun, C. P. Quantum coherence in multipartite systems. Phys. Rev. A 92, 022112 (2015).

20. Radhakrishnan, C., Parthasarathy, M., Jambulingam, S. \& Byrnes, T. Distribution of Quantum Coherence in Multipartite Systems. Phys. Rev. Lett. 116, 150504 (2016).

21. Bromley, T. R., Cianciaruso, M. \& Adesso, G. Frozen Quantum Coherence. Phys. Rev. Lett. 114, 210401 (2015).

22. Yu, X. D., Zhang, D. J., Liu, C. L. \& Tong, D. M. Measure-Independent Freezing of Quantum Coherence. Phys. Rev. A 93, 060303(R) (2016).

23. Ma, J., Yadin, B., Girolami, D., Vedral, V. \& Gu, M. Converting Coherence to Quantum Correlations. Phys. Rev. Lett. 116, 160407 (2016).

24. Xi, Z., Li, Y. \& Fan, H. Quantum coherence and correlations in quantum system. Sci. Rep. 5, 10922 (2015).

25. Chitambar, E. \& Hsieh, M. H. Relating the Resource Theories of Entanglement and Quantum Coherence. Phys. Rev. Lett. 117, 020402 (2016).

26. Chitambar, E. et al. Assisted Distillation of Quantum Coherence. Phys. Rev. Lett. 116, 070402 (2016).

27. Streltsov, A., Adesso, G., Huelga, S. F. \& Plenio, M. B. Colloquium: Quantum Coherence as a Resource. arXiv preprint arXiv:1609.02439 (2016).

28. Linden, N., Popescu, S. \& Smolin, J. A. Entanglement of superpositions. Phys. Rev. Lett. 97, 100502 (2006).

29. Gilad, G. Reexamination of entanglement of superpositons. Phys. Rev. A 76, 052320 (2007).

30. Niset, J. \& Cerf, N. J. Tight bounds on the concurrence of quantum superpositions. Phys. Rev. A 76, 042328 (2007).

31. Akhtarshenas, S. J. Concurrence of superpositions of many states. Phys. Rev. A 83, 042306 (2011).

32. Yue, Q. L. et al. Coherence of Superpositions. arXiv preprint arXiv:1605.04067 (2016).

33. Liu, F. \& Li, F. The number of terms in the superpositions upper bounds the amount of the coherence change. Quantum Inf. Process. 15, 4203 (2016).

34. Winter, A. \& Yang, D. Operational resource theory of coherence. Phys. Rev. Lett. 116, 120404 (2016).

35. Singh, U., Bera, M. N., Misra, A. \& Pati, A. K. Erasing Quantum Coherence: An Operational Approach. arXiv preprint arXiv:1506.08186 (2015).

36. Knill, E. \& Laflamme, R. Theory of quantum error-correcting codes. Phys. Rev. A 55, 900 (1997).

37. Audenaert, K. M. A sharp continuity estimate for the von Neumann entropy. J. Phys. A 40(28), 8127 (2007).

38. Hill, S. \& Wootters, W. K. Entanglement of a pair of quantum bits. Phys. Rev. Lett. 78, 5022 (1997).

\section{Acknowledgements}

W.Z. appreciates the financial support from China's 1000-Talent Plan, Q.Y., F.G. and Q.W. acknowledge the financial support from NSFC (Grants Nos 61572081, 61672110 and 61671082). Q.Y. and W.Z. thank the discussion with Eric Chitambar, W.Z. thanks the discussion with Barry C. Sanders.

\section{Author Contributions}

Q.Y., F.G. and W.Z. did the proof. Q.Y. wrote code and did the simulations. Q.Y., F.G., Q.W. and W.Z. wrote the manuscript. All authors discussed about the proof, reviewed and approved the final manuscript.

\section{Additional Information}

Competing Interests: The authors declare that they have no competing interests.

Publisher's note: Springer Nature remains neutral with regard to jurisdictional claims in published maps and institutional affiliations.

Open Access This article is licensed under a Creative Commons Attribution 4.0 International License, which permits use, sharing, adaptation, distribution and reproduction in any medium or format, as long as you give appropriate credit to the original author(s) and the source, provide a link to the Creative Commons license, and indicate if changes were made. The images or other third party material in this article are included in the article's Creative Commons license, unless indicated otherwise in a credit line to the material. If material is not included in the article's Creative Commons license and your intended use is not permitted by statutory regulation or exceeds the permitted use, you will need to obtain permission directly from the copyright holder. To view a copy of this license, visit http://creativecommons.org/licenses/by/4.0/.

(C) The Author(s) 2017 